\title{
Extração de Regras Fuzzy por meio da Aprendizagem de Máquina na Informática na Educação
}

\author{
Alana M. de Morais ${ }^{1}$, Aline M. de Morais² \\ ${ }^{1}$ Coordenação de Sistemas - IESP Faculdades, Centro Universitário De João Pessoa - \\ Unipê 2 \\ João Pessoa - PB - Brazil \\ alanam2@gmail.com, alinemmmcefet@gmail.com
}

\begin{abstract}
The evaluation of learners' achievement is an important phase in the learning in online courses. However, an assessment process may emerge in subjective terms (e.g., Good, Bad). Regards the uncertainty, the fuzzy logic is an appropriate approach for such cases because it can model the imprecise knowledge. The paper approach focuses on guide to construct the fuzzy rules by classification techniques (Decision Tree). The present study runs a validation using the FuzzySD approach, which aims to predict the student dropout phenomena in online courses. In this process, the teacher agreed with $70 \%$ of the rules generated by the analysis trees and obtained 19 fuzzy rules. Finally, when asked which the most intuitive tree for interpretation was, the specialist highlighted J48 type.
\end{abstract}

Resumo. No ensino online, a etapa de avaliação discente fornece informações que apoia o tomador de decisão em suas ações no ambiente. No entanto, a análise pode envolver critérios subjetivos (Bom ou Ruim). Diante desta incerteza, a lógica fuzzy é uma abordagem útil para esses casos, pois pode modelar o conhecimento impreciso. Este artigo se concentra em guiar a construção de regras difusas por meio de técnicas visuais de classificação de dados (árvores de decisão) com a ajuda do especialista na Informática na Educação. O presente estudo realiza uma validação utilizando a arquitetura da abordagem FuzzySD, sistema de predição de evasão discente em cursos online. Neste processo, o docente concordou com $70 \%$ das regras geradas pelas árvores de decisão que analisou, gerando 19 regras fuzzy. Por fim, ao ser questionado qual foi a árvore mais intuitiva à interpretação o especialista destacou o tipo $\mathrm{J} 48$.

\section{Introdução}

A avaliação do desempenho dos alunos é uma fase importante durante o processo de ensino e aprendizagem em cursos online. De acordo com os resultados obtidos nesta fase, diversas ações são executadas pelo tomador de decisão, tais como: reflexão sobre qualidade dos materiais pedagógicos, monitoramento do progresso da classe ou aluno e resoluções de problemas diante de situações inesperadas.

Os Ambientes Virtuais de Ensino e Aprendizagem (AVEA) estão cada vez mais apoiando tais análises por meio de ferramentas ou da liberação do acesso aos arquivos de $\log$ do curso para o tomador de decisão. Os arquivos de $\log$ registram todas as interações executadas pelos membros do curso em um determinado período. Contudo, 
VII Congresso Brasileiro de Informática na Educação (CBIE 2018)

Anais dos Workshops do VII Congresso Brasileiro de Informática na Educação (WCBIE 2018)

extrair informações deste tipo de estrutura não é uma tarefa trivial e rápida para o docente, pois exige um conhecimento técnico para o qual muitos deles não foram treinados.

Uma técnica muito presente nas avaliações educacionais é a lógica fuzzy, pois esta consegue lidar com entradas nebulosas ou difusas (Bom, Ruim, Correto, Errado, etc.). Esta técnica surge como um método de raciocínio com expressões lógicas que descreve a pertinência em conjuntos difusos. A ideia dos graus de pertinência possibilita agrupar os elementos de maneira diferente da aplicada na lógica clássica, o que permite reinterpretar antigos conceitos, elaborados segundo esta lógica [Russell e Norvig, 2013].

De maneira geral, o sistema lógico fuzzy consiste em três etapas principais: fuzzificação, inferência e defuzzificação. O presente estudo foca seus esforços na construção das regras importantes para a fase da inferência, especificamente, na automatização da extração das regras fuzzy, de acordo com uma determinada base de dados. Para isto, o trabalho validou a abordagem por meio dos dados do arcabouço FuzzySD [Morais, 2017], focado na predição da evasão discente em cursos online sob o formato da UAB (Universidade Aberta do Brasil). Este trabalho considera evasão quando um aluno permanece sem acessar e interagir com o ambiente online por um período ininterrupto maior do que um quarto do tempo de execução do curso online.

Técnicas de Aprendizagem de Máquina, especificamente classificação, foram utilizadas para atingir os objetivos definidos pelo estudo. Como principal contribuição, o presente artigo pode destacar a discussão e validação de uma estratégia para extração de regras realizada de modo intuitivo e gráfico, o que facilita o envolvimento e participação do especialista na área da Informática na Educação.

A fim de esclarecer o método científico utilizado e os resultados do estudo, o presente artigo foi organizado em 5 seções principais. Na segunda seção, discutiu-se trabalhos que almejaram definir estratégias para automatizar a geração de regras fuzzy. $\mathrm{Na}$ terceira seção deste artigo, o planejamento e execução do nosso estudo de caso foram detalhados e discutidos. Além disto, a quarta seção contemplou os principais resultados das investigações. Por fim, a quinta e última seção apresentou as considerações finais e os trabalhos futuros relacionados ao estudo.

\section{Sistemas baseados em Lógica Fuzzy}

De maneira geral, o sistema lógico fuzzy consiste em três operações principais: fuzzificação, inferência e defuzzificação lógica [Russell e Norvig, 2013]. Inicialmente, na etapa de fuzzificação, o problema deve ser analisado e os dados de entrada transformados em variáveis linguísticas. Neste momento, é de extrema importância que todos os dados de incerteza sejam analisados e transformados em variáveis linguísticas. Após isto, são determinadas também as funções de pertinência para cada uma das entradas definidas na arquitetura fuzzy. No segundo momento, a etapa de inferência deve ser iniciada. Nesta fase, são criadas as regras ou proposições por meio da associação das variáveis já definidas. Por fim, a etapa de defuzzificação envolve a conversão dos valores fuzzy em números reais, tendo assim um conjunto de saída matematicamente definido.

Como mencionado anteriormente, o entendimento das etapas fuzzy não é uma tarefa trivial para a maioria dos tomadores de decisão da Educação a Distância (EaD), principalmente o processo de geração de regras. As regras são estruturas construídas por 
VII Congresso Brasileiro de Informática na Educação (CBIE 2018)

Anais dos Workshops do VII Congresso Brasileiro de Informática na Educação (WCBIE 2018)

meio de condições "SE-ENTÃO" (SE característica= x ENTÃO saída= z). Contudo, um sistema fuzzy eficiente requer ou um especialista no tema para ajustá-las ou um algoritmo de classificação supervisionado que possibilite extrair tais regras. Nesta segunda alternativa, pode-se fazer uso de um método de aprendizado que absorva conhecimento da base de dados e o expresse.

\subsection{Geração de Regras Fuzzy}

Neste sentido, alguns trabalhos surgem com o intuito de apoiar os tomadores de decisão em contextos distintos para automatizar o processo de geração de regras de arquiteturas fuzzy. Diversas técnicas podem ser utilizadas com este intuito e a presente seção almeja citar algumas delas. Assim, destacam-se as técnicas de Aprendizagem de Máquina, área da computação que tem o intuito de identificar padrões de comportamento e automatizar a construção de modelos analíticos. É um ramo da Inteligência Artificial baseado na ideia de que sistemas podem aprender com dados, identificar padrões e tomar decisões.

É possível destacar abordagens que combinam os métodos de agrupamento com outros mecanismos de aprendizado para a geração de regras fuzzy. Por exemplo, a combinação de Algoritmos Genéticos e Agrupamentos pode ser encontradas em Saez et al. (2008). Em Lee et al. (2008) é apresentado um novo algoritmo de agrupamento fuzzy iterativo que incorpora um esquema de supervisão em uma abordagem de agrupamento fuzzy não supervisionado. $O$ processo de aprendizado começa de modo não supervisionado e constrói partições significativas no espaço de entrada. As regras fuzzy são geradas por meio de uma seleção de grupos com supervisão baseado nas noções de pureza e separabilidade dos grupos [Stanovov et al. 2015].

A análise de agrupamento passou a ser explorada como um importante mecanismo para geração de regras de um sistema fuzzy, em função do crescimento do interesse por técnicas que permitam a construção automática de tais sistemas a partir de conjuntos de dados. A abordagem mais comum para gerar regras por meio de métodos de agrupamento fuzzy é considerar cada grupo como uma possível regra.

Outra estratégia relevante é a utilização de mapas de auto-organização (SelfOrganizing Maps - SOM) para extrair parâmetros de regras fuzzy automaticamente sem intervenção humana. Esta estrutura funciona bem em conjuntos de dados de pequena escala com dezenas de milhares de amostras. O trabalho de Shalaginov e Franke (2015) estudou a aplicabilidade e a melhoria do método original de Kosko para análise de dados em larga escala, incluindo despejos de tráfego de rede com várias centenas de milhares de amostras no contexto da avaliação de mensagens maliciosas por meio de uma rede neuro-fuzzy.

Uma abordagem distinta pode ser observada no trabalho de Semenkin e Stanovov (2014), que utiliza um algoritmo evolucionário auto-configurável para automatizar a base de regras fuzzy analisada. Este método é muito parecido com o Algoritmo Genético, que é frequentemente usado para formar sistemas difusos, mas tem a capacidade de ajustar o número e o comprimento das regras, possui operadores especiais de crossover e inicialização e a parte de Michigan. A parte de Michigan do algoritmo permite o ajuste preciso de bases de regras para o problema de classificação usando objetos classificados incorretamente para a construção de novas regras. Também exclui as regras que descrevem apenas uma pequena parte da amostra para simplificar a base de regras. O algoritmo pode encontrar bases de regras precisas e pequenas 
VII Congresso Brasileiro de Informática na Educação (CBIE 2018)

Anais dos Workshops do VII Congresso Brasileiro de Informática na Educação (WCBIE 2018)

rapidamente, e seu desempenho é comparável a outros métodos conhecidos.

\section{2. Árvores de Decisão}

Existem muitos algoritmos de aprendizado que induzem classificadores. Nesta seção, são descritos indutores que contribuem e priorizam o entendimento dos dados. Por exemplo, no desenvolvimento de sistemas especialistas é primordial que especialistas humanos possam verificar de modo rápido e fácil o conhecimento extraído e relacionálo ao seu próprio domínio de conhecimento.

Uma das técnicas utilizadas neste contexto são as árvores de decisão. Entende-se por árvores de decisão as estruturas formadas por um conjunto de nós de decisão, perguntas, que permitem a classificação de cada caso [Russell e Norvig, 2013]. Uma árvore de decisão é definida recursivamente como [Nilsson, 2014]: um nó folha que corresponde a uma classe ou um nó de decisão que contém um teste sobre algum atributo. Para cada resultado do teste existe uma aresta para uma subárvore.

Outra relevância das árvores de decisão, é que sua facilidade de compreensão contribui para o entendimento do domínio considerado, fato que pode produzir um conhecimento novo para o especialista. O tempo de geração do modelo é menor em comparação ao de outras técnicas e, na maioria das vezes, não necessita de informações adicionais. As árvores de decisão são modelos estatísticos, que podem ser usados com o objetivo de classificar objetos. Neste tipo de aplicação, a partir de um conjunto de instâncias, cujas classes são conhecidas, após a fase de treinamento, uma árvore de decisão é capaz de classificar novas instâncias. As árvores de decisão foram utilizadas para auxiliar na classificação e automatização no processo de geração de regras fuzzy.

Há diversas técnicas que podem ser utilizadas, sendo a pioneira delas a ID3. O algoritmo ID3, cuja criação se baseou em sistemas de inferência e em conceitos de sistemas de aprendizagem, foi um dos primeiros de árvore de decisão. Logo após, foram elaborados diversos outros algoritmos, sendo os mais conhecidos o C4.5, o J48, a AD Tree, a NBTree, o CART (Classification and RegressionTrees) e o CHAID (Chi Square Automatic Interaction Detection), além de outros.

\section{Metodologia}

Esta seção tem como intuito apresentar e discutir o método científico utilizado (estudo de caso) e o cenário selecionado durante a extração das regras fuzzy analisadas.

\subsection{Estudo de Caso}

Para avaliar a metodologia proposta, foi selecionado um sistema fuzzy no contexto da Informática na Educação como estudo de caso. A extração de regras foi executada por meio do uso das árvores de decisão no contexto da abordagem FuzzySD [Morais 2017]. A abordagem avaliativa mencionada tem como propósito analisar os indicadores multidimensionais associados às atividades pedagógicas do aluno no ambiente (Interação, Motivação e Autorregulação) e, com isto, prover contribuições à avaliação dos discentes por meio do monitoramento de métricas que descrevam suas interações nos cursos online. Este monitoramento visa predizer o fenômeno da evasão em cursos online no formato UAB. O presente trabalho considera evasão quando um aluno permanece sem acessar ou interagir com o ambiente online por um período ininterrupto maior do que um quarto do tempo de execução do curso online. 
VII Congresso Brasileiro de Informática na Educação (CBIE 2018)

Anais dos Workshops do VII Congresso Brasileiro de Informática na Educação (WCBIE 2018)

No FuzzySD, foi executada a etapa de fuzzificação e as regras de pertinência foram construídas, não existem regras definitivas na metodologia fuzzy para a escolha de tais funções que representam o conhecimento de um especialista no assunto em questão ou informações extraídas de um banco de dados. Algumas justificativas e testes fizeram com que o estudo convergisse a definir a função de pertinência do tipo triangular e trapezoidal como a mais adequada para as entradas da abordagem apresentada dentre as quais: a aplicação em pesquisas correlatas sobre avaliação multidimensional e concordância por parte do docente consultor. A funções de pertinência foram analisadas e planejadas em conjunto com o professor mediante a uma análise das métricas e de dados históricos relacioanados a cada uma delas.

Para realizar este experimento, foram utilizados dados das interações dos alunos do $1^{\circ}$ período de Letras do IFPB Virtual do período 2015.2. A amostra analisada nesta análise de correlação contou com 148 alunos matriculados e uma taxa de evasão foi de $35 \%$ (número de alunos evadidos/número de alunos matriculados). $\mathrm{O}$ estudo de caso foi conduzido de forma offline, o que significa que as análises são executadas a posteriori ao curso online. Além disto, todos os participantes tiveram o mesmo ambiente e tempo para interagir com as atividades propostas.

\subsection{Arcabouço FuzzySD}

O FuzzySD é um arcabouço avaliativo que tem como propósito analisar os indicadores multidimensionais associados às atividades pedagógicas do aluno no ambiente e, com isto, prover contribuições à avaliação dos discentes por meio do monitoramento de métricas que descrevam suas interações nos cursos online. Tal investigação é útil no acompanhamento do perfil evasivo dos usuários no AVEA. A Figura 1 apresenta a arquitetura do arcabouço utilizado.

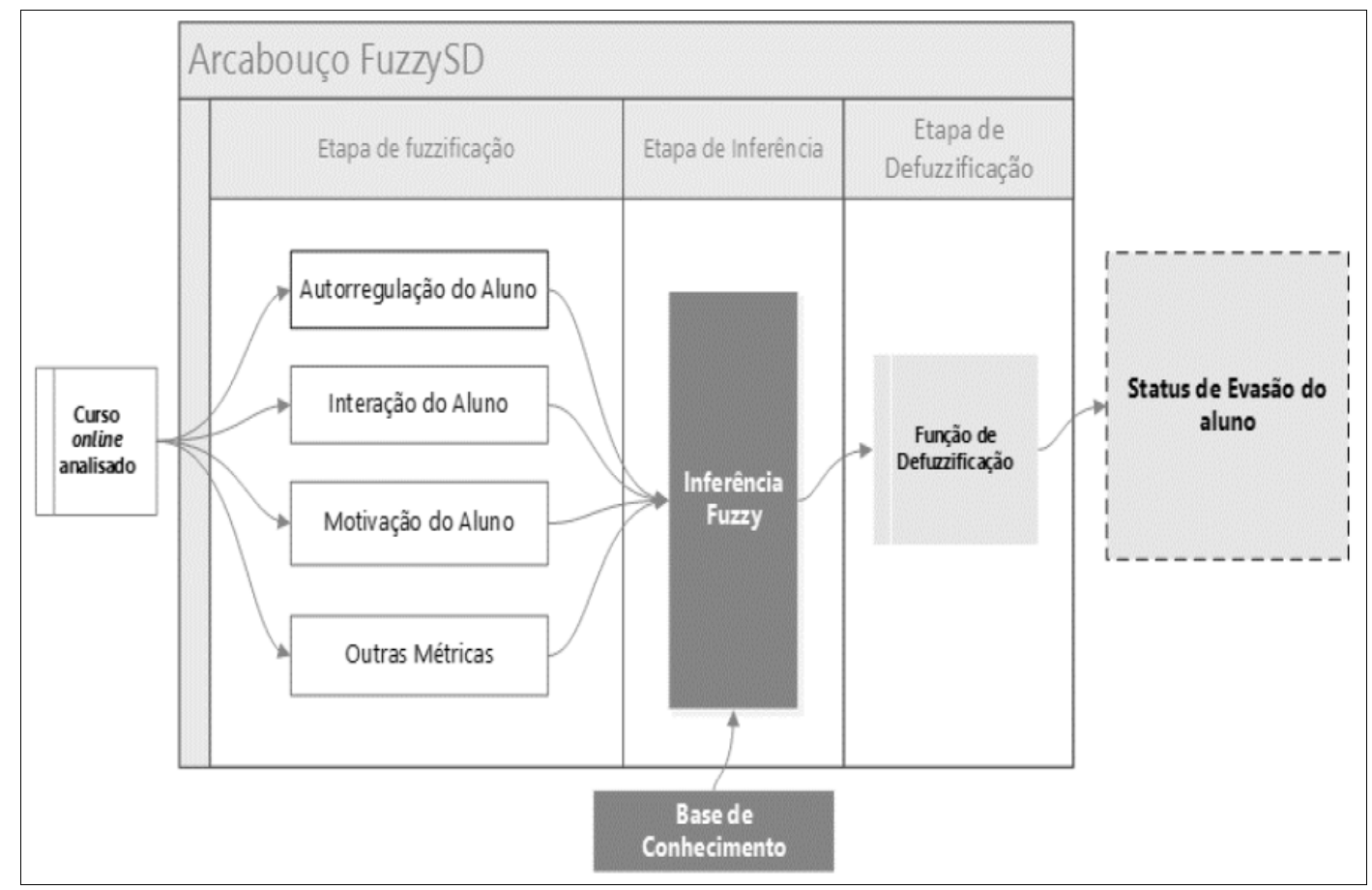

Figura 1. Arcabouço FuzzySD [Morais 2017].

Cada curso tem suas peculiaridades e cada professor seus critérios de avaliação, que dependem da sua visão de mundo, de sociedade e da educação. Por isto, é 
VII Congresso Brasileiro de Informática na Educação (CBIE 2018)

Anais dos Workshops do VII Congresso Brasileiro de Informática na Educação (WCBIE 2018)

necessário elencar alguns indicadores que são parâmetros para que o professor adapte seus objetivos ao arcabouço proposto.

$\mathrm{O}$ arcabouço analisado tem como entradas algumas métricas de comportamento discente, a saber: Autorregulação do aluno, Interação do aluno, e Motivação do aluno. Tais medidas são imprecisas, pois envolvem variáveis fuzzy (Autorregulação: "Autorregulado", "Em Autorregulação", "Não Autorregulado"; Motivação: "Participativo", "Pouco Participativo", "Não Participativo"; Interação: "Desmotivado", "Motivado Extrinsecamente", "Motivado Intrínseca").

\subsection{Extração das Regras}

A partir do método de inferência, ou seja, utilizando o conhecimento dos especialistas para desenvolver um raciocínio, modelam-se as incertezas dentro de um processo para tomada de decisão, fundamentado na existência de conexões entre causa e efeito, por meio de regras, naturais ou não, denominadas regras de inferência.

É importante ressaltar que tais regras condicionais podem ser inseridas no contexto da base de conhecimento. Caso um especialista julgue necessário adicionar alguma informação posterior à modelagem, regras como modificadores linguísticos, condições, proposições poderão ser inseridas no modelo a fim de tornar o sistema ainda mais robusto.

Neste caso, as regras foram definidas e depois examinadas de maneira distinta. No processo de inferência, os princípios da lógica fuzzy foram usados para combinar as regras fuzzy "SE-ENTÃO" existentes na base de regras com os dados fuzzy de entrada. O resultado desta combinação representou uma região fuzzy de saída que se relacionada com a saída do processo. Os passos para o alcance dessa região fuzzy de saída foram constituídas por: (i) definir as regras que estejam ativadas em um determinado momento; (ii) determinar a saída fuzzy de cada uma das regras ativadas; e (iii) combinar todas as saídas fuzzy calculadas.

A definição dos termos verbais, funções de pertinência e a inferência da base de regras, provenientes da teoria da lógica fuzzy, foram etapas executadas em sequência e resultaram na geração do modelo matemático do arcabouço.

A base de conhecimento é composta pela base de regras e pela base de dados. A base de dados armazena as definições das funções de pertinência, bem como as definições de normalização e discretização dos universos de discurso. A base de regras possui o conjunto de regras de produção que apresentam a estrutura SE <premissa> ENTÃO <consequente>.

O módulo de inferência processa os dados de entrada e as regras de produção inferindo as ações de controle nebulosas de acordo com o estado do sistema. É necessário definir o conjunto de regras pertencentes à base de conhecimento, com isso, há uma regra para cada termo linguístico de entrada multiplicados entre si, resultando em 19 regras condicionais associadas aos seus respectivos termos de saída. Tais regras foram obtidas por meio de conversas com o professor especialista e, principalmente, por meio da aprendizagem de regras extraídas por meio de técnicas de classificação, especificamente as árvores de decisão. As árvores de decisão do tipo Random Tree, NBTree, ADTree e $J 48$ permitiram a identificação de regras que inicialmente o especialista não conseguiu perceber em uma análise pontual. 
VII Congresso Brasileiro de Informática na Educação (CBIE 2018)

Anais dos Workshops do VII Congresso Brasileiro de Informática na Educação (WCBIE 2018)

\section{Resultados}

Com base na metodologia proposta, foram executados os algoritmos definidos e gerados diversas árvores sobre as interações no estudo de caso. Após a geração destas estruturas, uma reunião com o professor consultor foi realizada para que as regras fossem analisadas.

As conversas eram entrevistas semiestruturadas, ou seja, tópicos foram definidos, mas a discussão era livre e não precisava ser delimitada pelo entrevistador. Nestas conversas, quatro tipos de árvores foram utilizados: Random Tree, NBTree, ADTree e J48. Tais algoritmos foram selecionados dada a sua relevância na literatura e os resultados obtidos em outros estudos. Outro aspecto importante para a seleção de tais técnicas foi a sua facilidade de interpretação, dado as saídas que foram analisadas pelo professor para analisar as regras. A proposta de utilizar árvores é apoiar o especialista a planejar as regras se houver dados históricos no contexto.

\subsection{Exemplo de Árvore de Decisão Gerada}

A Figura 2 e Figura 3 ilustram exemplos de algumas árvores apresentadas para o docente durante a entrevista. Em ambas as elipses representa as métricas fuzzy do arcabouço (Autorregulação, Interação e Motivação) e nas linhas pode-se observar as variáveis linguísticas associadas a cada uma das métricas. Os nós finais (ou folhas), representadas pelos retângulos das árvores representam as saídas finais do modelo relacionado à métrica de saída Evasão (Evadido ou Persistente, no caso da árvore do algoritmo J48).

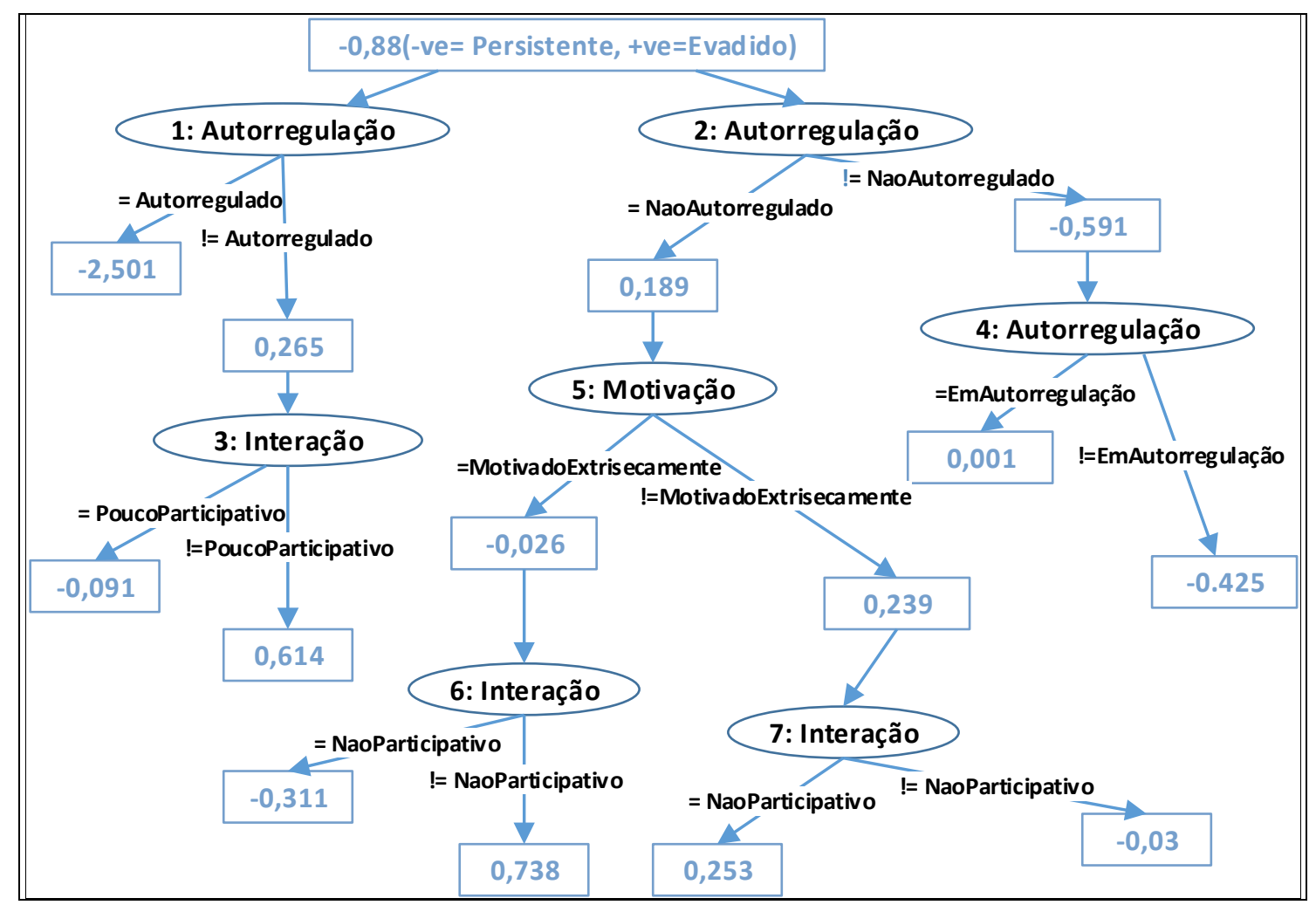

Figura 2. Exemplo de Árvore ADTree.

Após algumas instruções sobre a sintaxe da árvore, o docente iniciou o processo 
VII Congresso Brasileiro de Informática na Educação (CBIE 2018)

Anais dos Workshops do VII Congresso Brasileiro de Informática na Educação (WCBIE 2018)

de extração das regras a partir das árvores de decisão. A Figura 4 ilustra um exemplo de leitura que foi feita pelo professor. As palavras em vermelho exemplificam o sentido da leitura realizada. A árvore de decisão J48 diz ao professor que um aluno que tenha a métrica Autorregulação como "Não Autorregulado", a Interação como "Pouco Participativo" e a Motivação como "Desmotivado" provavelmente este aluno terá o status de Evadido ao final do curso segundo o comportamento da amostra anterior analisada. Neste caso, o professor concordou com a regra e a adicionou à lista de regras que compõe a base de conhecimento do arcabouço FuzzySD.

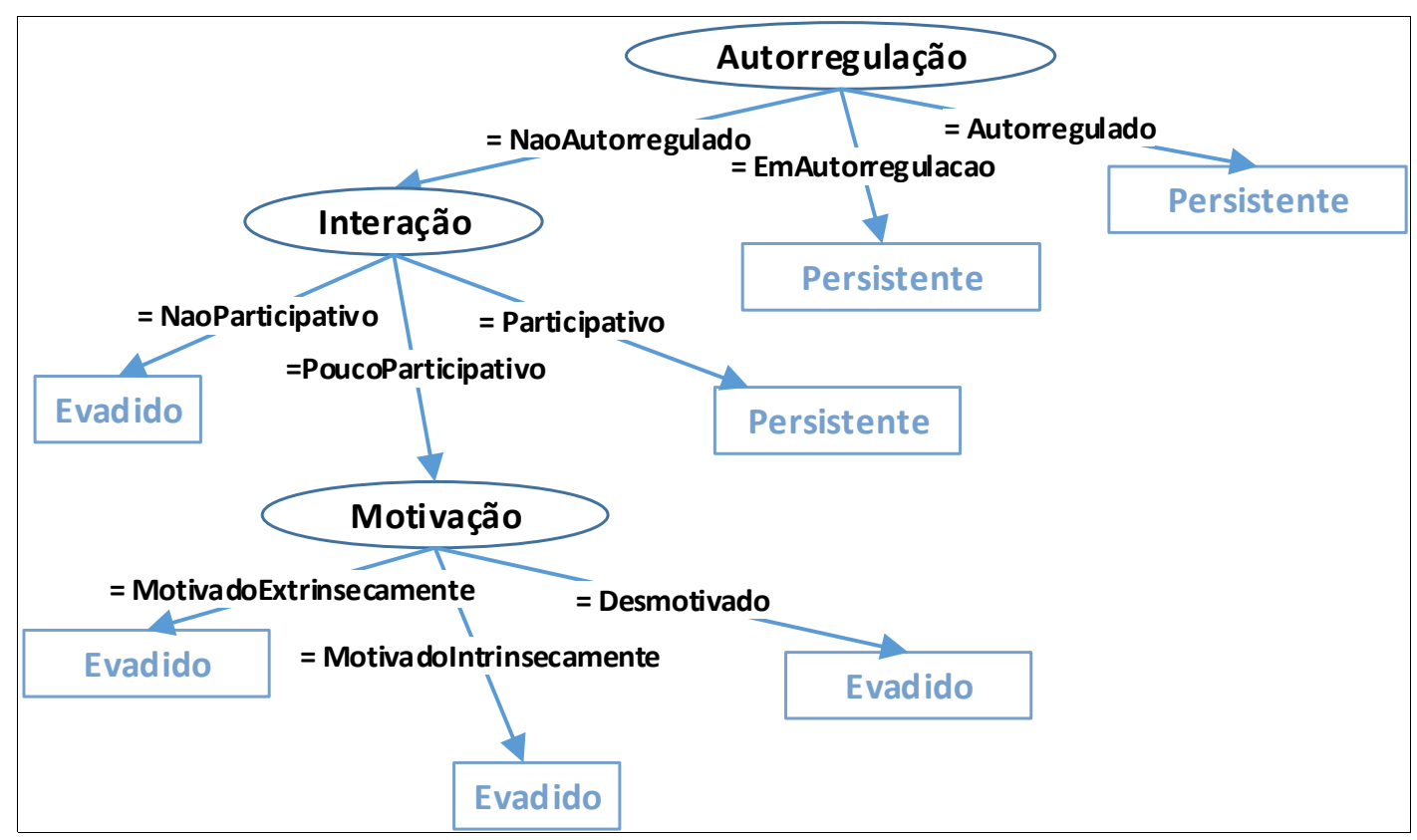

Figura 3. Exemplo de Árvore J48.

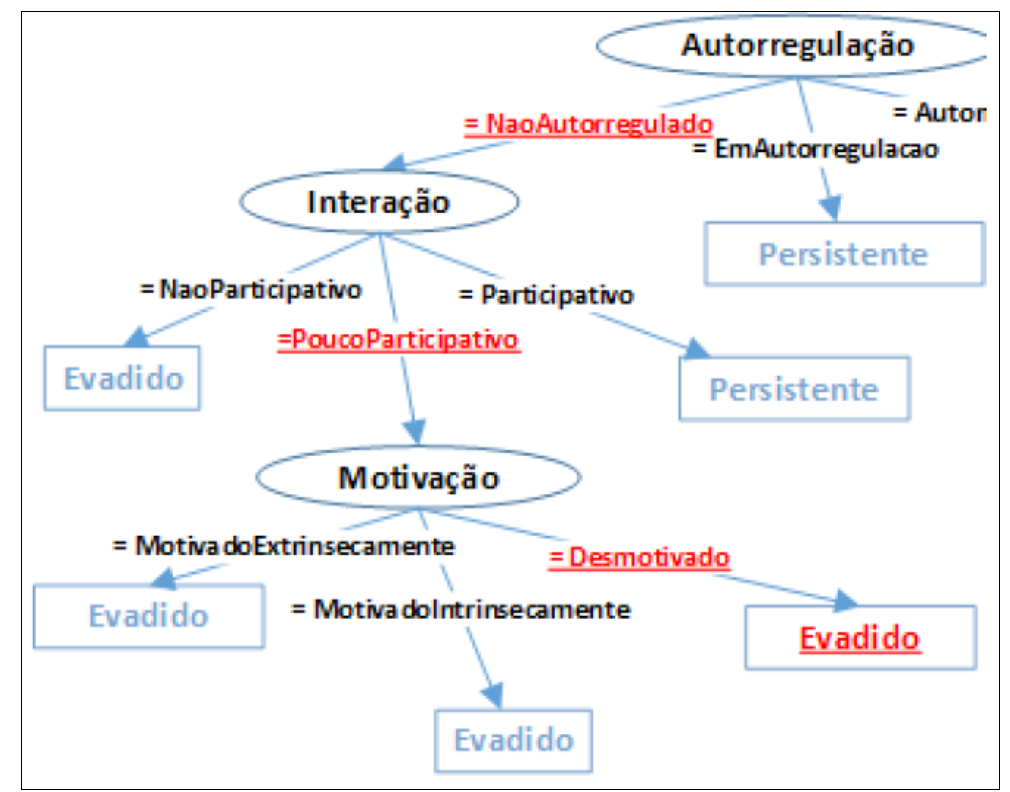

Figura 4. Exemplo de extração de regra pelo docente.

Este tipo de análise se repete inúmeras vezes até que ao final diversas regras sejam analisadas e criadas. Caso o professor não concorde com a regra ou haja um 
VII Congresso Brasileiro de Informática na Educação (CBIE 2018)

Anais dos Workshops do VII Congresso Brasileiro de Informática na Educação (WCBIE 2018)

confronto de informações entre as árvores, o especialista sempre terá a opinião final sobre a regra.

\subsection{Regras Extraídas}

Foram geradas um total de 27 regras, no qual 10 regras destas representavam situações ambíguas em que entradas iguais tinham saídas diferentes dependendo da árvore analisada. Ao final, obteve-se uma regra para cada termo linguístico de entrada, multiplicados entre si, além de diversas outras combinações, resultando em 19 regras condicionais associadas aos seus respectivos termos de saída (Tabela 1). Tais regras foram obtidas por meio das conversas mencionadas com o professor especialista e, principalmente, por meio da aprendizagem de regras extraídas por meio de técnicas de classificação, especificamente as árvores de decisão. Este professor convidado por ser o responsável pela disciplina em análise.

Ao longo do processo, o docente concordou com $70 \%$ das regras geradas pelas árvores que analisou. Ao ser questionado qual foi a árvore mais intuitiva à interpretação o docente destacou a árvore do tipo $\mathrm{J} 48$.

Tabela 1. Regras fuzzy do sistema FuzzySD

\begin{tabular}{|l|l|l|l|l|}
\hline Regra & Autorregulação & Interação & Motivação & Evasão \\
\hline $\mathbf{1}$ & Autorregulado & Participativo & Motivado Intrinsecamente & Persistência \\
\hline $\mathbf{2}$ & Autorregulado & Participativo & Motivado Extrinsecamente & Persistência \\
\hline $\mathbf{3}$ & Autorregulado & Pouco Participativo & Motivado Intrinsecamente & Persistência \\
\hline $\mathbf{4}$ & Autorregulado & Não Participativo & Desmotivado & Evasão \\
\hline $\mathbf{5}$ & Autorregulado & Não Participativo & Motivado Intrinsecamente & Persistência \\
\hline $\mathbf{6}$ & Em Autorregulação & Pouco Participativo & Motivado Extrinsecamente & Persistência \\
\hline $\mathbf{7}$ & Em Autorregulação & Participativo & Motivado Extrinsecamente & Persistência \\
\hline $\mathbf{8}$ & Em Autorregulação & Não Participativo & Motivado Extrinsecamente & Persistência \\
\hline $\mathbf{9}$ & Em Autorregulação & Pouco Participativo & Motivado Intrinsecamente & Evasão \\
\hline $\mathbf{1 0}$ & Em Autorregulação & Participativo & Desmotivado & Persistência \\
\hline $\mathbf{1 1}$ & Em Autorregulação & Pouco Participativo & Desmotivado & Persistência \\
\hline $\mathbf{1 2}$ & Não Autorregulado & Participativo & Desmotivado & Persistência \\
\hline $\mathbf{1 3}$ & Não Autorregulado & Pouco Participativo & Desmotivado & Evasão \\
\hline $\mathbf{1 4}$ & Não Autorregulado & Não Participativo & Motivado Extrinsecamente & Evasão \\
\hline $\mathbf{1 5}$ & Não Autorregulado & Não Participativo & Motivado Intrinsecamente & Persistência \\
\hline $\mathbf{1 6}$ & Não Autorregulado & Pouco Participativo & Motivado Intrinsecamente & Evasão \\
\hline $\mathbf{1 7}$ & Não Autorregulado & Pouco Participativo & Motivado Extrinsecamente & Evasão \\
\hline $\mathbf{1 8}$ & Não Autorregulado & Participativo & Motivado Extrinsecamente & Persistência \\
\hline $\mathbf{1 9}$ & Não Autorregulado & Pouco Participativo & Desmotivado & Persistência \\
\hline
\end{tabular}

\section{Considerações Finais}

Neste artigo, foi proposto uma metodologia para a extração de regras fuzzy em contextos complexos por meio do uso de árvores de decisão. Significativamente, o método tenta encontrar um conjunto compacto de regras difusas usando técnicas de classificação de 
VII Congresso Brasileiro de Informática na Educação (CBIE 2018)

Anais dos Workshops do VII Congresso Brasileiro de Informática na Educação (WCBIE 2018)

dados.

Durante o processo de construção de um arcabouço fuzzy baseado em regras, sobretudo em contextos complexos, podem existir inúmeras regras igualmente boas para modelar o problema, pois o número de regras diferentes e combinações possíveis entre regras pode ser extremamente elevado. Entretanto, somente algumas poucas delas podem ser compatíveis com a semântica que o especialista espera para o modelo.

A estratégia de utilizar as árvores de decisão foi relevante no contexto de cursos online, pois permitiu que o professor entendesse mais claramente domínios pouco explorados e pudesse interferir mais ativamente na construção das regras e na construção da base de conhecimento da arquitetura. A principal limitação da abordagem adotada foi a necessidade de dados históricos para a construção das árvores discutidas.

Este trabalho contemplará como próximas etapas uma investigação da eficiência das árvores de decisão em relação aos Algoritmos Genéticos e Clusterizações. Outra atividade que será contemplada pelos autores é uma investigação sobre estratégias não supervisionada para contextos de cursos online.

\section{Referências Bibliográficas}

Ken, F. et al. (2010) "A Tree-Based Decision Model to Support Prediction of the Severity of Asthma Exacerbations in Children". Journal of Medical Systems, v.34, p.551-562.

Lee, H. E.; Park, K. H. e Bien, Z. Z. (2008) "Iterative fuzzy clustering algorithm with supervision to construct probabilistic fuzzy rule base from numerical data". IEEE Transactions on Fuzzy Systems. v. 16, n. 1, p. 263-277.

Morais, A. (2017). Planejamento de um Arcabouço Avaliativo para a Identificação do Perfil Evasivo do Discente: Estudo de Caso IFPB Virtual. In Anais do Workshop de Informática na Escola (Vol. 23, No. 1, p. 993-1002).

Nilsson, N.J. (2014). Principles of artificial intelligence. Morgan Kaufmann.

Russell, S. e Norvig, P. (2013) "Inteligência Artificial" (3ª Edição). ISBN: 978-85-3523701-6. Rio de Janeiro: Elsevier, 2013.

Saez, Y.; Zazo, F. e Isasi, P. (2008). "A study of the effects of clustering and local search on radio network design: Evolutionary computation approaches." In: Eighth International Conference on Hybrid Intelligent Systems p. 951-954.

Semenkin, E. e Stanovov, V. (2014) "Fuzzy rule bases automated design with selfconfiguring evolutionary algorithm". In Informatics in Control, Automation and Robotics (ICINCO), 2014 11th International Conference on (Vol. 1, pp. 318-323). IEEE.

Shalaginov, A. e Franke, K. (2015) "Automated generation of fuzzy rules from largescale network traffic analysis in digital forensics investigations." In Soft Computing and Pattern Recognition (SoCPaR), 2015 7th International Conference of (pp. 3136). IEEE.

Stanovov, V., Sopov, E. e Semenkin, E. (2015) "Multi-strategy multimodal genetic algorithm for designing fuzzy rule based classifiers." In Computational Intelligence, 2015 IEEE Symposium Series on (pp. 167-173). IEEE. 